\title{
Correlation between Follicular Fluid's Androgen Level and Fertilization Rate in Poor Responder Patients Undergone IVF: A Prospective Cohort Done in Yasmin IVF Clinic, Jakarta, Indonesia
}

\section{Budi Wiweko ${ }^{1}$, Nadia Shafira ${ }^{1}$, Kresna Mutia², Anom Bowolaksono ${ }^{3}$, Muharam Natadisastral, and Andon Hestiantoro}

1Department of Obstetrics and Gynecology, Faculty of Medicine, Universitas Indonesia, Jakarta, Indonesia

${ }^{2}$ Indonesian Reproductive Medicine Research and Training Centre, Jakarta, Indonesia

${ }^{3}$ Department of Biology, Faculty of Mathematics and Natural Sciences, Universitas Indonesia, Depok, Banten, Indonesia

\section{Abstract}

Introduction. Androstenedione (A4), testosterone ( $T$ ), and dehidroepiandrosterone (DHEA) are known to be involved in folliculogenesis and follicular maturity. Lower follicular androgen levels in poor responder due to malfunctioned granulosa and

Corresponding Author: Nadia Shafira; email:

nadia_shafira@hotmail.com

Received: 24 August 2016 Accepted: 25 September 2016 Published: 4 October 2016

Publishing services provided by Knowledge E

(c) Budi Wiweko et al. This article is distributed under the terms of the Creative

commons Attribution

License, which permits unrestricted use and redistribution provided that the original author and source are credited.

Selection and Peer-review under the responsibility of the ASPIRE Conference Committee.

\section{G OPEN ACCESS} theca cells and decreased inhibin B production will decrease FSH and LH. However, androgenic follicular fluid might also induce follicular atresia, decreased oocytes viability, thus affecting fertilization. The aim of current study is to find the correlation between intra-follicular androgen levels and fertilization rate, specifically in poor responder whom contributed in $84 \%$ cancelled cycle. Material and Methods. This prospective cohort study was done at Yasmin IVF Clinic, Jakarta, Indonesia, in January 2014-March 2015. Infertile women undergone IVF were asked to participate, grouped into poor responder and other, and the androgen levels in the follicular fluid of each consenting patients were measured. Results. From total 62 patients, aged $23-47$ years old $(37.6 \pm 5,068)$, there were 21 poor responders, whereas the other 41 patients with other indication. In poor responder group, levels of androstenedione, testosterone, and DHEA were 50.8 - 272.3 (103.5 \pm 59.9), 383.2 - 1747.9 (1114.4 \pm 373.2$), 11.3-$ $454.8(151.3 \pm 96.8)$, whereas in other group the androgen levels were $44.3-326.8$ $(95.1 \pm 61.2), 414.1-1463.7(976.9 \pm 258.4), 44.6-265.8$ (132.7 \pm 61.3$)$ with the correlation with fertilization rate $0.609,0.095$, and 0.361 respectively. Conclusion. Correlation between follicular androgen levels and fertilization rate found to be not significantly different. The low number of subjects might cause this result, as well as the presence of bias, e.g.male factor and endometriosis might also affect fertilization. A multi-center study with larger sample size added with thorough analysis is needed to reconfirm current data.

Keywords: In Vitro Fertilization, Follicular Fluid, Androgen Level, Poor Responder, Oocyte Quality, Fertilization Rate 


\section{Introduction}

Androstenedione (A4), testosterone ( $T$ ), and dehidroepiandrosterone (DHEA) are 3 androgens known to be involved in folliculogenesis. These hormones trigger FSH activity for granulosa cells' development and differentiation, which in turn, leads to follicular maturity. There were several studies reporting lower follicular androgen levels in poor responder due to malfunctioned granulosa and theca cells added with decreased inhibin B production causing decreased FSH and LH. However, it was also found the androgenic follicular fluid might induce follicular atresia, decreased oocytes viability, thus affecting fertilization $[1,2,4]$.

Inconsistencies of previous data looking for the correlation between intra-follicular androgen levels and the oocyte quality, specifically in poor responder whom contributed in $84 \%$ cancelled cycle, is the background of this study to be arranged.

\section{Material and Methods}

\subsection{Time and Place of Study}

This prospective cohort study was done at Yasmin IVF Clinic, dr. Cipto Mangunkusumo Hospital, Jakarta, Indonesia, between January 2014-March 2015.

\subsection{Subjects of Study}

Infertile women undergone IVF were asked to participate and the follicular fluid of each consenting patients were collected and measured for its A4, T, and DHEA levels. Those women were grouped into 2, poor responder and other, which are women with PCOS, endometriosis and adenomyosis, male factor, and unexplained infertility The poor responder group should matched minimum 2 of these following criteria, 1) advanced age ( $\geq 40$ years old) or with other risk factor for poor responder; 2 ) history of poor response on the previous cycles with less than 3 oocytes picked up after conventional protocol; and 3) poor ovarian reserve (antral follicle count (AFC) less than 5-7 follicles or AMH level less than 0.5-1.1 ng/ml).

\subsection{Data Analysis}

The correlation between the androgen levels and fertilization rate was then analyzed using SPSS 11 program.

\section{Results}

From total 62 patients recruited in the study, with the youngest was 23 years old and the oldest is 47 years old (mean $37.6 \pm 5,068$ ), there were 21 poor responder patients, whereas the other 41 patients were classified into other indication group. Number 
of mature follicle, oocytes retrieved, and the 2pn cell of the fertilization day in poor responder group was $2-18(5.14 \pm 3.65), 1-3(2.19 \pm 0.81)$, and $1-2(1.5 \pm 0.42)$, respectively; whereas the range number in other group was 4 - 38 (14.15 \pm 9.49$)$, $4-24(8.95 \pm 5.85)$ and $2-13(5.5 \pm 3.72)$ respectively. In poor responder group, levels of androstenedione, testosterone, and DHEA were 50.8 - 272.3 (103.5 \pm 59.9 ), $383.2-1747.9$ (1114.4 \pm 373.2$), 11.3-454.8(151.3 \pm 96.8)$, whereas in other group the androgen levels were $44.3-326.8$ (95.1 \pm 61.2$), 414.1-1463.7$ (976.9 \pm 258.4$), 44.6$ $-265.8(132.7 \pm 61.3)$ with the correlation with fertilization rate $p$ value $0.609,0.095$, and 0.361 respectively.

These results showed there were no significant difference of the androgen levels between poor responder patients and patients with other indications, related to the number of mature follicles produced and the fertilization rate, although both groups share similar characteristics of subjects. Both groups shown similar baseline androgen levels and giving a similar rate of fertilization.

There were limited studies looking at the androgen levels within the follicular fluid on IVF patients. Follicular fluid is the environment for a follicle to grow, therefore the hormone levels within its fluid will represent its optimal condition to produce the best mature follicles, and thus, best oocytes quality [1-4]. Intra-follicular androgen levels are useful to be determined, as it can be used as the baseline data for supplementation therapy of androgen for IVF patients with poor ovarian reserve or with history of poor IVF response [5-8].

Current result has reversed with previous study done by Revelli et al (2009) [2] reporting that higher androgen levels were related to a lower oocytes quality and cleavage rate. Moreover, this result also contradict with studies done by Jonard et al on 2003 [9] and Frattarelli and Peterson (2004) [6] showing that the low serum level of testosterone, androstenedione and DHEA were related to the poorer IVF outcome.

Even though there was a theory about androgenic intra-follicular environment may lead to follicle atresia, it was also known that a certain value of intra-follicular androgen levels needed for an optimal growth of follicles $[1,4,10]$. Wen et al (2010) [10] stated that a higher level of steroids will be found on follicles with more mature oocytes and better fertilization rate. However, de Los Santos et al on 2013 had a disagreement with the previous statement that they reported poor responders and good responders has no different follicular androgen levels [5].

There were limitations on this study. The first limiting factor would be the variety of contributing factor to bias the result of the study, such as the sperm quality or the endometriosis factor which might effect the follicular growth itself as well as the fertilization rate. Moreover, the androgen levels were measured in the time of oocytes retrieval after the ovarian stimulation was done, therefore the actual level of baseline androgen cannot be evaluated. The presence of serum hormonal baseline levels data might also give a better report if these data can be analyzed in the study as a multifactorial analysis to decrease the bias. The number of subjects recruited as well as the singular place of recruitment might also limit the generalization of the data provided. A broader and larger multi center study with a multifactorial analysis will give a better result whilst reconfirming the available data provided by current study. 


\begin{tabular}{l|ll} 
Subjects $(n=62)$ & Poor Responder $(n=21)$ & Other $(n=41)$ \\
Overall Age Range & $23-47$ years old $(37.6 \pm 5.068)$ \\
Age Range each Group & $\begin{array}{l}33-47 \text { years old }(40.4 \pm 3.72) \\
23-42 \text { years old }(36.1 \pm \\
5.068)\end{array}$ \\
$\begin{array}{l}\text { Type of Infertility } \\
\text { Primary Infertility }\end{array}$ & 19 & 24 \\
Secondary Infertility & 2 & 17
\end{tabular}

TABLE 1: Subjects characteristics.

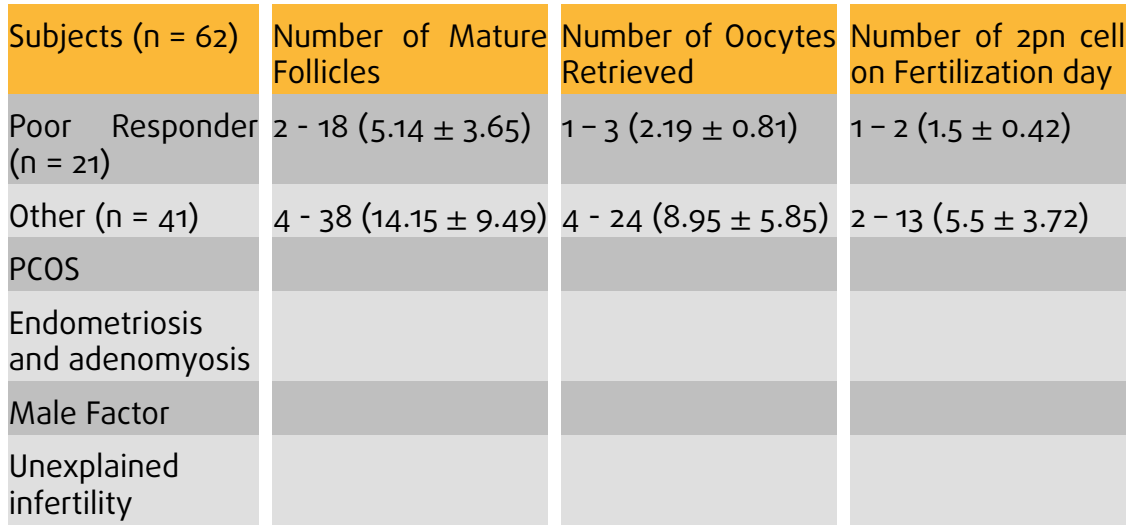

TABLE 2: Mature follicles, oocytes retrieved, and 2pn cell on fertilization day.

\subsection{Conclusion}

Correlation between androgen levels on follicular fluid and fertilization rate found to be not significantly different between the two groups. The low number of subjects might cause this result, as well as the presence of bias due to male factor and endometriosis might also affect fertilization. A multi-center study with larger sample size added with thorough analysis of multiple factors related to the fertilization rate is needed to reconfirm current data.

\section{References}

[1] S. M. Nelson, Biomarkers of ovarian response: Current and future applications, Fertility and Sterility, 99, no. 4, 963-969, (2013).

\begin{tabular}{|c|c|c|c|}
\hline $\begin{array}{l}\text { Androgens Level in Follicular } \\
\text { Fluid }\end{array}$ & Poor Responder $(n=21)$ & Other $(n=41)$ & $\begin{array}{l}\text { Correlation with } \\
\text { Fertilization Rate }\end{array}$ \\
\hline Androstenedione (ng/dl) & $50.8-272.3(103.5 \pm 59.9)$ & $44.3-326.8(95.1 \pm 61.2)$ & $p 0.609$ \\
\hline Testosterone (pg/mL) & $\begin{array}{l}383.2-1747.9(1114.4 \pm \\
373.2)\end{array}$ & $\begin{array}{l}414.1-1463.7(976.9 \pm \\
258.4)\end{array}$ & \\
\hline DHEA $(\mu \mathrm{g} / \mathrm{mL})$ & $11.3-454.8(151.3 \pm 96.8)$ & $44.6-265.8(132.7 \pm 61.3)$ & $p 0.361$ \\
\hline
\end{tabular}

TABLE 3: Androgen levels and its correlation with fertilization rate. 
[2] A. Revelli, L. D. Piane, S. Casano, E. Molinari, M. Massobrio, and P. Rinaudo, Follicular fluid content and oocyte quality: From single biochemical markers to metabolomics, Reproductive Biology and Endocrinology, 7, article no. 40, (2009).

[3] K.-H. Tsui, L.-T. Lin, R. Chang, B.-S. Huang, J.-T. Cheng, and P.-H. Wang, Effects of dehydroepiandrosterone supplementation on women with poor ovarian response: A preliminary report and review, Taiwanese Journal of Obstetrics and Gynecology, 54, no. 2, 131-136, (2015).

[4] K. A. Walters, Role of androgens in normal and pathological ovarian function, Reproduction, 149, no. 4, R193-R218, (2015).

[5] M. J. De Los Santos, V. García-Laez, D. Beltrán, E. Labarta, J. L. Zuzuarregui, P. Alamá, P. Gámiz, J. Crespo, E. Bosch, and A. Pellicer, The follicular hormonal profile in lowresponder patients undergoing unstimulated cycles: Is it hypoandrogenic? Human Reproduction, 28, no. 1, 224-229, (2013).

[6] J. L. Frattarelli and E. H. Peterson, Effect of androgen levels on in vitro fertilization cycles, Fertility and Sterility, 81, no. 6, 1713-1714, (2004).

[7] N. Gleicher, D. H. McCulloh, V. A. Kushnir, N. Ganguly, D. H. Barad, K. N. Goldman, M. M. Kushnir, D. F. Albertini, and J. A. Grifo, Is there an androgen level threshold for aneuploidy risk in infertile women? Reproductive Biology and Endocrinology, 13, no. 1, article no. 38, (2015).

[8] L.-T. Lin, K.-H. Tsui, and P.-H. Wang, Clinical application of dehydroepiandrosterone in reproduction: A review of the evidence, Journal of the Chinese Medical Association, 78, no. 8, 446-453, (2003).

[9] S. Jonard, Y. Robert, C. Cortet-Rudelli, P. Pigny, C. Decanter, and D. Dewailly, Ultrasound examination of polycystic ovaries: Is it worth counting the follicles? Human Reproduction, 18, no. 3, 598-603, (2003).

[10] X. Wen, D. Li, A. J. Tozer, S. M. Docherty, and R. K. Iles, Estradiol, progesterone, testosterone profiles in human follicular fluid and cultured granulosa cells from luteinized pre-ovulatory follicles, Reproductive Biology and Endocrinology, 8, article no. 117, (2010). 